Caraban B.M. ${ }^{3}$, Romila Aurelia ${ }^{2}$, Hangan L.T. ${ }^{3}$, Lungu Mihaela ${ }^{1}$

\title{
Cerebral Vasospasm in Subarachnoid Hemorrhage Through Aneurysm Rupture - Clinical Considerations and Case Report
}

${ }^{1}$ Neurological Department, Emergency Clinical Hospital, Galati; Faculty of Medicine and Pharmacy, "Dunarea de Jos" University of Galati

${ }^{2}$ Geriatric Department, Emergency Clinical Hospital, Galati; Faculty of Medicine and Pharmacy, "Dunarea de Jos" University of Galati, Center for Research on Medical-Pharmaceutical

${ }^{3}$ Faculty of Medicine, University "Ovidius" of Constanta

\begin{abstract}
Arterial aneurysm ruptures of the circle of Willis determine subarachnoid

hemorrhage, which evolving due to the irritating effect of the blood in the subarachnoid space may lead to complications such as large arterial vasospasm in the origin of the large cerebral arteries, from the base of the brain.

Cerebral vasospasm causes a downstream cerebral ischemia, that may lead to the establishment of an ischemic stroke which is life threatening.

Early treatment against the vasospasm with calcium channels blockers should prevent occurrence of ischemia. However, the effectiveness of this treatment is not fully confirmed, fact that was mentioned even in the presentation of our case.
\end{abstract}

Keywords: subarachnoid hemorrhage, aneurysm rupture, vasospasm, cerebral ischemia, calcium blockers.

\section{Bogdan Marian Caraban}

Faculty of Medicine, University "Ovidius" of Constanta University Street, No. 1, Campus B, Constanta, Romania

email : bcaraban@yahoo.com

phone: +40722322476

\section{Introduction}

Subarachnoid hemorrhage is the fourth cerebrovascular disease as frequency, after atherothrombosis, embolism and primary intracerebral hemorrhage.

The incidence for subarachnoid hemorrhage is highest between the ages of 35 and 65 years old, in the US registering 26000 cases/ year.

One of the mechanisms of developing subarachnoid hemorrhage is the rupture of saccular aneurysm with origin in the arteries of the circle of Willis.

Aneurysms of the circle of Willis arteries occur because of development of disorders in the arterial wall, with alteration of the medial tunic and of the elastic one or through focal destruction of the internal elastic membrane under the influence of hemodynamic forces acting on the arterial bifurcations. As a result, the intimate tunic bulges outside covered only by the adventitia. The sacciform aneurysm that occurs gradually increases and eventually it breaks.

Aneurysm sizes range from $2 \mathrm{~mm}$ to $2-3 \mathrm{~cm}$ in diameter; generally it seems that the aneurysms that 
break are with a diameter of over $10 \mathrm{~mm}$, although the breach may occur in even smaller aneurysms.

The aneurysm rupture site is in the aneurysmal dome, which can present one or more secondary saccular dilatations.

Aneurysm localization: $90-95 \%$ of them are located in the anterior area of the circle of Willis, originating in:

- anterior communicating artery;

- the origin of the posterior communicating artery from the internal carotid artery;

- the first major bifurcation of the middle cerebral artery;

- bifurcation of the internal carotid artery into the anterior cerebral artery and middle cerebral artery.

Other localizations of aneurysms include: internal carotid artery in the cavernous

sinus, the origin of the ophthalmic artery, the junction between the posterior communicating artery and the posterior cerebral artery, basilar artery bifurcation.

Aneurysmal rupture leads to subarachnoid hemorrhage whereas when the rupture occurs the blood which is under pressure is forced to enter the subarachnoid space which leads to symptoms such as: violent headache, vomiting, with or without loss of consciousness.

A severe complication of subarachnoid hemorrhage, caused by the existence of blood in the subarachnoid space is the arterial vasospasm.

The vasospasm is a major cause of death and disability in patients with subarachnoid hemorrhage, being responsible for $50 \%$ of the deaths occurring in this severe condition.

The vasospasm occurs in $70 \%$ of patients with subarachnoid hemorrhage, and about $30 \%$ of the survivors develop a delayed ischemic neurological deficit, which may have regressive development or which may lead to stroke up called symptomatic cerebral vasospasm.

The vasospasm has multiple causes, some factors being the products of hemolyzed blood seeping inward from the pia-arachnoid into the muscularis of the artery.

Because of the vasospasm, the arterial wall undergoes structural changes - progressive proliferative vasculopathy, in a chronic process that affects since the days 1-8 the adventitia, media and partly the intimate membrane. Smooth muscle fibers of the middle membrane necroses, the adventitia is infiltrated with neutrophils, mast cells and red blood corpuscles, some of which have migrated to a subendothelial position.

Due to the vasospasm, the decrease of arterial perfusion in the arterial affected territory occurs with the apparition of a cerebral stroke area - confirmed through imaging, in the territory of some arteries affected by the spasm and which do not have atherosclerosis lesions/ thrombosis.

Thus ischemic lesions that form are often multiple.

Brain damage produced by the spasm and thus neurological signs that appear depend on the affected arterial territory ( ex. Aphasia, hemiparesis), which may create confusion with the occurrence of some symptoms caused by the acute hydrocephalus secondary to the subarachnoid hemorrhage.

The vasospasm occurrence can be detected by transcranial Doppler which detects increased blood flow of more than $175 \mathrm{~cm} / \mathrm{s}$, the examination being imposed in days $4,7,10,14,17$ and 23 , and for patients with Hunt Hess score 3-5 daily [4].

Clinically, the spasm onset is in the first 3-5 days of the occurrence of the subarachnoid hemorrhage, when the maximum arterial narrowing appears to take place in the days 5-8 (6-8), followed by a gradual return with a maximum duration of 2-4 weeks, on average 18-21 days.

The vasospasm therapy tries to decrease the risk of neural damage by controlling the intracranial hypertension, decreasing the metabolic rate of oxygen consumption, improving cerebral circulation.

Thus, the clinician can reach out to the following therapeutic options:

1. Medication that reduces vasospasm, including:

1.1 Calcium channel blockers

Nimodipine - is a dihydropyridine calcium channel blocker acting on smooth

muscle fibers from the arterial wall which would reduce mortality rate and improve prognosis for the patient with vasospasm that occurred after subarachnoid hemorrhage. American Association 
Guide for Stroke recommends nimodipine in doses of $16 \mathrm{mg}$ every 4 hours, while monitoring the blood pressure (which will be maintained at values of $\sim 160 \mathrm{mmHg}$ systolic) over a period of 21 days. There are also authors who state that $30 \mathrm{mg}$ of nimodipine every 2 hours would have a better clinical outcome, especially in patients with low blood pressure. Nimodipine can be administrated - in progressive vasospasm with a clinical stable condition - intraarterial or intra-venous: IV $5 \mathrm{~mm} / \mathrm{h}$ at a sufficient level of blood pressure over 190/110mmHg [4]. There are some studies which state that IV administration of nimodipine in vasospasm treatment is not superior to oral administration, but it increases the risk of orthostatic hypotension, which imposes bed rest. The effect can be appreciated by transcranial Doppler.

It is the only approved drug by the FDA in the vasospasm treatment, having a half-life of approximately 9 hours. Studies have demonstrated that nimodipine improves the angiographic aspect of the vasospasm, reducing the risk of ischemia secondary to subarachnoid hemorrhage, having a good safety profile [7].

However, the mechanism trough which nimodipine acts on the vasospasm is still unclear, which imposes further study in this regard.

Nicardipine is a second generation dihydropyridine - type $\mathrm{CCB}$, with possible neuroprotective preventive effect, that selectively inhibits the movement of calcium within the smooth muscular fibers [3]. It also has an antihypertensive effect, as well as regional selection in the smooth muscles and cerebral arteries. Huang et al. state that nicardipine reduces the risk of death trough vasospasm in patients with subarachnoid hemorrhage trough aneurysm rupture. However its use remains controversial due to its side effects: pulmonary edema, kidney failure, prolonged hypotension.

Verapamil, used mainly in the treatment of coronary spasm, has not proven effective in vasospasm that occurs after subarachnoid hemorrhage.

\subsection{Statins}

Statins are considered HMG-CoA reductase inhibitors, which appears to assign an important role in preventing vasospasm. There are clinical studies which focused on pravastatin and simvastatin administrated for vasospasm and which showed a reduction of the arterial narrowing with reduced risk of secondary ischemia [10]. But there are also studies that have not shown any benefit after administrating there drugs, therefore the study of the statin benefit in vasospasm treatment remains open.

1.3 Phosphodiesterase inhibitors

Milrinone acts on c-AMP having inotropic and vasodilation effect, studies conducted in 2001 showing that it would have a significant effect in reducing vasospasm in subarachnoid hemorrhage [2]. The drug was administrated in association with nimodipine, but the secondary effects with an important decrease of the blood pressure limits its use.

Cilostazol is included in the antiplatelet group, inhibiting the phosphodiesterase action on the platelets and the smooth muscle fibers with vasodilation effect. There are studies show that cilostazol significantly reduces the risk of vasospasm ischemia, but it induces tachycardia and action its action mechanism is not fully understood which imposes as well further trials.

1.4 Endothelin agonists

Clazosentan has pros and cons studies over the effect of reducing vasospasm in aneurysmal ruptures and in this case further studies being required.

\subsection{Hormones}

For now there were studies conducted on animals with erythropoietin which have shown that it would have a neuroprotective role in symptomatic vasospasm.

Estrogens have vasodilation, anti-inflammatory and neuroprotective effects but are still undergoing studies on laboratory pets to prove their effectiveness in symptomatic vasospasm treatment.

1.6 Fasudil

Fasudil is a Rho- kinase inhibitor with effect on the inhibition of the protein phosphorylation, studied in the case of vasospasm prevention from extracranial artery stenting [5]. Liu Jian Guang et.al. conducted studies that demonstrated that fasudil has significantly reduced vasospasm in subarachnoid hemorrhage, but the low number of trials can't demonstrate yet its efficiency and safety.

\subsection{Nitric oxide NO}

Nitric oxide seems to be involved in the development of symptomatic vasospasm, its reduction in the blood and the cerebral spinal fluid being the two mechanisms possibly involved. However in this case 
as well there are further clinical trials required [8].

2. Avoiding hypovolemia, maintaining a normal volume of circulating blood: treatment " $3 \mathrm{H}$ ".

- Hypertension can be corrected with 100$1500 \mathrm{micrograms} / \mathrm{min}$ of dopamine or $0,6-0,8 \mathrm{mg} / \mathrm{h}$ of arterenol for systolic blood pressure values of $160-200 \mathrm{mmHg}$.

- Hemodilution - hypervolemia for which we may administrate human albumin $5 \%, 250 \mathrm{ml} \times 3$ per day or HAES (hydroxyethilstarke) $6 \%, 1000 \mathrm{ml}$ per day for optimal values of the central nervous pressure of $6-10 \mathrm{mmHg}$.

3. Transluminal balloon angioplasty could be considered a therapeutic option of

vasodilation in situations of the affecting of the big arteries within the circle of Willis, this statement still needing to be checked throughout further clinical studies.

However, following these therapeutic steps offers no guarantee of eliminating the risk of vasospasm occurrence, a fact confirmed by presenting the next clinical case of aneurysm rupture and subarachnoid hemorrhage complicated by arterial vasospasm and the occurrence of an ischemic stroke, that occurred despite early and proper administration of the antispasmodic treatment.

\section{Case Report}

Patient G.A. of age 62 was admitted to the neurology service presenting violent acute headache, nausea, vomiting that debuted in full health. Objectively, there was a syndrome of meningeal irritation detected with marked neck stiffness.

The patient did not have any cardiovascular risk factors and in personal history there were not found any impairments of vascular cerebral disorders, coronarian diseases or any disorders involving peripheral arteries in the lower limbs.

The brain CT detected blood in the subarachnoid space, predominantly in the anterior interhemispheric fissure and it suggested the presence of an aneurysm possibly belonging to the anterior communicating artery - Figures. 1,2.

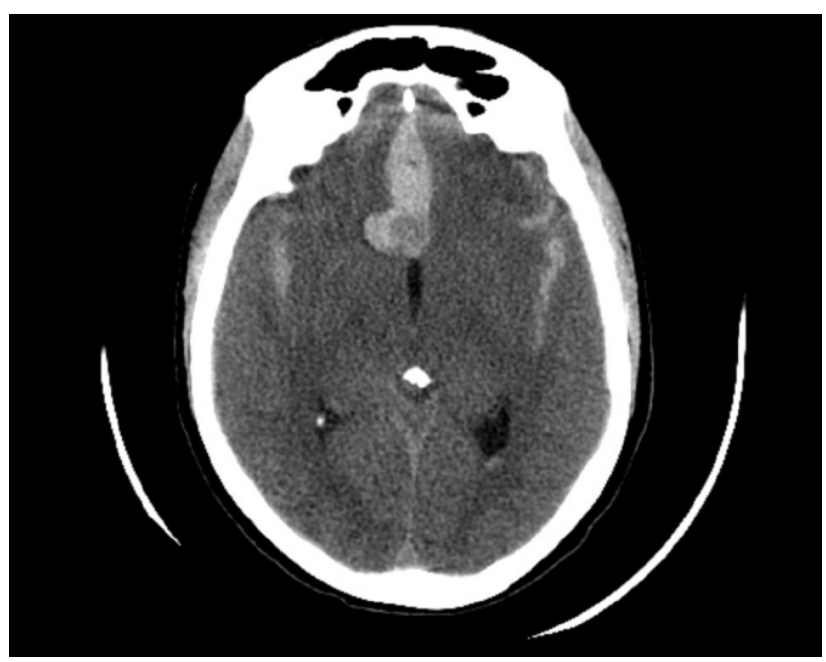

Figure 1 Subarachnoid hemorrhage in the anterior interhemispheric fissure with a possible anterior cerebral artery aneurysm.

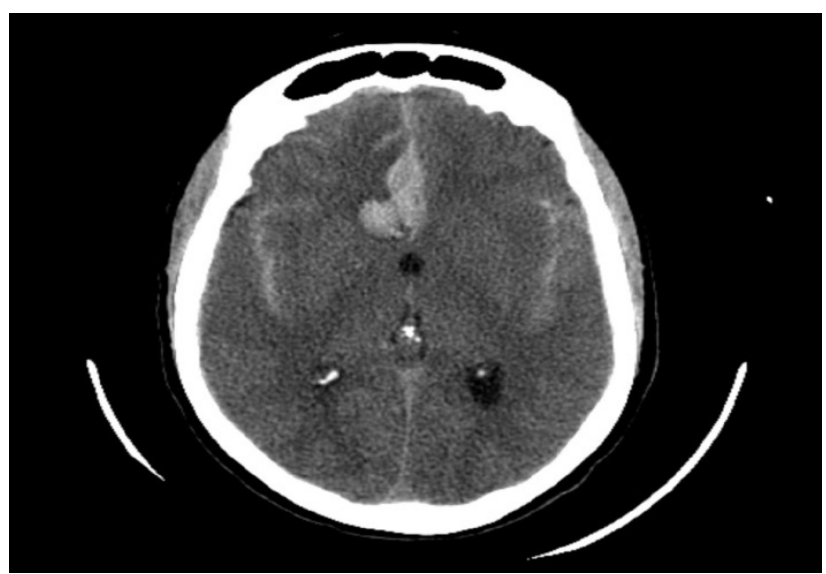

Figure 2 Aspect of subarachnoid hemorrhage with a suspicion of anterior cerebral artery aneurysm.

Oral prophylaxis is started for the cerebral vasospasm secondary to the subarachnoid hemorrhage with nimodipine: $60 \mathrm{mg}$ every 4 hours. Blood pressure was maintained throughout the treatment at values of $160 / 90 \mathrm{mmHg}$.

With a suspected rupture of anterior cerebral 
artery aneurysm and secondary subarachnoid hemorrhage - grade 2 on the Hunt Hess scale, the patient is transferred to a neurosurgery clinic where an angiographic examination is carried out on 4 vessels and it detects an anterior communicating artery aneurysm with a diameter of $40 \mathrm{~mm}$. We resort to surgical intervention for clipping the aneurysm followed up by postoperative angiographic control, showing a correct aneurysmal clipping - Figure 3 .

During this time we continue oral administration of nimodipine: $60 \mathrm{mg}$ every 4 hours, the patient does not show any blood volume disorders or changes of the blood pressure.

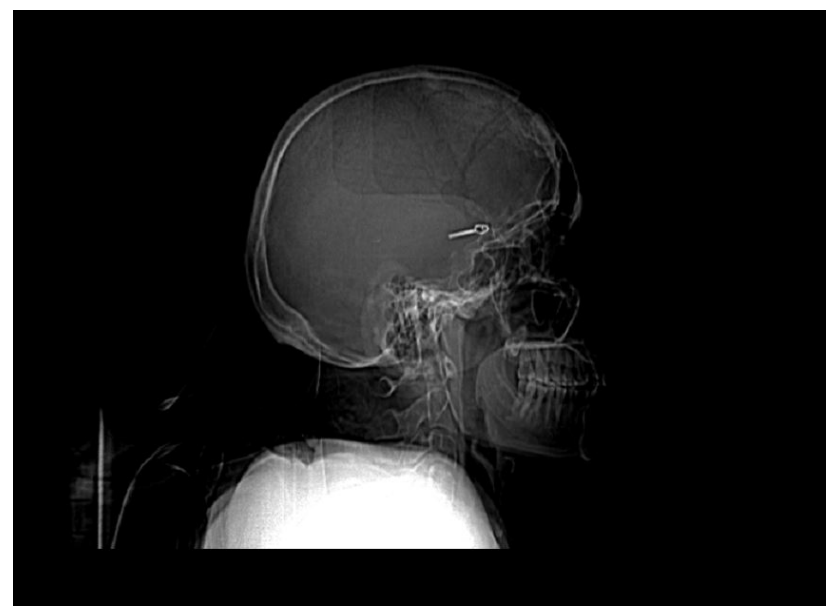

Figure 3 Anterior cerebral artery aneurysm clipping.

On the $11^{\text {th }}$ day after onset and continuously being under the prevention treatment against the vasospasm with the scheme mentioned previously, the patient starts to have a left motor deficit, crural plegia $\mathrm{MRC}=0 / 5$ and brachial palsy $\mathrm{MRC}=2 / 5$, associated with a frontal lobe apathy syndrome, bradypsychia, bradylalia, motional indifference gatism, left forced grasping reflex.

The patient's blood volume and blood pressure were stable.

She is readmitted into neurology where the brain CT is repeated and it detects bilateral frontal ischemia - in the area of the anterior cerebral artery, predominantly on the right side, the affected arterial territory being the one previously exposed to an intense subarachnoid bleeding - Figure 4.

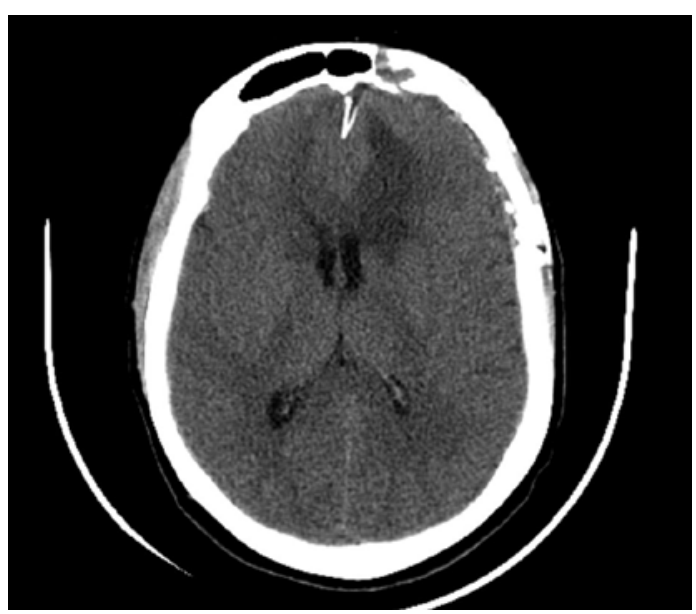

Figure 4 Aspect of frontal ischemia in the anterior cerebral artery area, predominantly on the right side.

\section{Discussions}

The clinical context and the imaging features support the diagnosis of multiple cerebral ischemia in the anterior cerebral artery area, predominantly on the right side, setting up an ischemic stroke, although the patient received throughout all the immediate period after the aneurysm rupture, a fair treatment against the vasospasm, having her blood volume and blood pressure stabilized.

In this case, the anterior cerebral artery was not affected by preexisting atherothrombotic processes, the patient did not have any cardiovascular accidents or any vascular risk factors.

The development of the motor deficit was slow but favorable towards improvement, but the frontal lobe syndrome was still persistent. 
The cerebral vasospasm is a severe complication of the subarachnoid hemorrhage,

life threatening for the patients who survived the subarachnoid hemorrhage.

This may lead to a cerebral stroke - sometimes multiple, with severe neurological deficits or even death and it may progress despite a correct treatment for its prevention.

The vasospasm may exceed in terms of aggression the direct effects of the aneurysm rupture or even of the bleeding.

The mechanism of the vasospasm's appearance it's not fully understood.

Early treatment is of major importance.

Although many therapeutic options are under trial, the only approved drug by the medical practice guides is nimodipine, the rest still needing to be confirmed or denied by the results of future trials.

However, the treatment with calcium blockers administrated according to the rules - has not proven it's effectiveness in combating the vasospasm in the patient whose case was presented.

In the presented case the vasospasm caused cerebral ischemia in the $11^{\text {th }}$ day after the subarachnoid bleeding and it could not be countered by the action of the administrated calcium blockers.

\section{Acknowledgment}

Financial disclosure: none.

Informed consent: Informed consent was obtained from the patient in this case report.
1. Ropper, A., Samuels, M. \& Klein, J. (2014). Adams and Victor's Principles of Neurology 10th Edition: McGraw-Hill Education (pp 778, 844853).

2. Arakawa, Y., Kikuta, K., Hojo, M., Goto, Y., Ishii, A. \& Yamagata, S. (2001). Milrinone for the treatment of cerebral vasospasm after subarachnoid hemorrhage: report of seven cases. Neurosurgery. 48(4), 723-728; discussion 728730.

3. Huang, R.-q., Jiang, F.-g., Feng, Z.-m. \& Wang, T.-y. (2013). Nicardipine in the treatment of aneurysmal subarachnoid haemorrhage: a metaanalysis of published data. Acta Neurologica Belgica. 113(1), 3-6. doi: 10.1007/s13760-0120142-x.

4. Hufschmidt, A. \& Lucking, C.H. (2002). Integral neurology from symptom to treatment; Ed. Polirom.

5. Liu, J., Yao, G.E., Zhou, H.D., Jiang, X.J. \& Xie, P. (2014). Clinical investigation of fasudil for the prevention of cerebral vasospasm in extracranial carotid artery stenting. Cell Biochem Biophys. 68(1), 185-188. doi: 10.1007/s12013-013-96874.

6. Liu, Y., Qiu, H., Su, J. \& Jiang, W. (2016). Drug Treatment of Cerebral Vasospasm after Subarachnoid Hemorrhage Following Aneurysms. Global Journal of Medical Research: A. Neurology and Nervous System. 16(1), 11-20.

7. Ott, S., Jedlicka, S., Wolf, S., Peter, M., Pudenz, C., Merker, P., Sch, \#xfc, rer, L. \& Lumenta, C.B. (2014). Continuous Selective Intra-Arterial Application of Nimodipine in Refractory Cerebral Vasospasm due to Aneurysmal Subarachnoid Hemorrhage. BioMed Research International. 2014, 11. doi: 10.1155/2014/970741

8. Sehba, F.A., Schwartz, A.Y., Chereshnev, I. \& Bederson, J.B. (2000). Acute decrease in cerebral nitric oxide levels after subarachnoid hemorrhage. J Cereb Blood Flow Metab. 20(3), 604-611. doi: 10.1097/00004647-200003000- 
00018

9. Simon, R.P., Greenberg, D. \& Aminoff, M.J. (1999). Clinical Neurology, Seventh Edition: McGraw-Hill Education. (pp. 82-86)

10. Vergouwen, M.D., de Haan, R.J., Vermeulen, M. \& Roos, Y.B. (2010). Effect of statin treatment on vasospasm, delayed cerebral ischemia, and functional outcome in patients with aneurysmal subarachnoid hemorrhage: a systematic review and meta-analysis update. Stroke. 41(1), e47-52. doi: 10.1161/STROKEAHA.109.55633 